\title{
Rulers of light
}

\author{
Frequency combs measure optical frequencies with an unprecedented precision, allowing myriad applications in \\ optical metrology, high-precision spectroscopy, optical atomic clocks, attosecond science, astronomy and, recently, \\ quantum information processing.
}

A $\mathrm{n}$ optical frequency comb is a light source with a spectrum containing not a continuum, but a discrete pattern of sharp, narrow, equidistant laser lines (pictured). It can be generated via a number of mechanisms, notably via the stabilization of a pulse train with a fixed repetition rate generated by a femtosecond mode-locked laser, or via four-wave mixing in nonlinear media. The former technique was developed by Theodor Hänsch and John Hall around 2000, which led to the duo sharing half of the 2005 Nobel Prize in Physics "for their contributions to the development of laser-based precision spectroscopy, including the optical frequency comb technique."

Functioning like a 'ruler' of light, these combs have allowed scientists to determine optical frequencies with great precision, revolutionizing fundamental science. For instance, the remarkable progress in frequency comb sources dedicated to broadband spectroscopy - especially in the mid-infrared region $(2-20 \mu \mathrm{m})$, used for molecular fingerprinting, and the ultraviolet range $(<400 \mathrm{~nm})-$ has opened up new opportunities for exploring atomic and molecular structures and dynamics. As with spectroscopy, frequency combs can support conventional laser ranging approaches by functioning as a precise spectral ruler. Frequency comb light sources have been used in optical clocks, enabling precise time keeping. In the time domain, the control of the relative phase between the envelope and the carrier of ultrashort pulses has enabled the study of dynamics that occur at a fraction of an optical cycle, a key to attosecond science. In recent years, a new regime of precision radial-velocity measurements in the search for Earth-like exoplanets has been enabled by high-resolution spectrographs calibrated by laser frequency combs, extending the use of these 'astrocombs' as a spectrographic observational tool in astronomy.

In this focus issue on frequency combs, we present a collection of three Review Articles that provide an update on the latest progress of on-chip generation of frequency combs, frequency comb spectroscopy, and the emerging role of frequency combs in quantum science and technology.

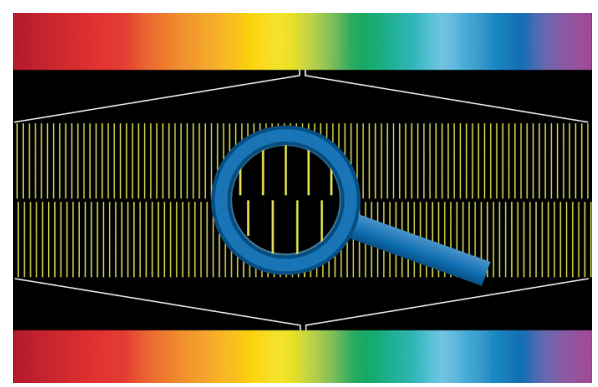

Credit: Theodor W. Hänsch and Nathalie Picqué, Max-Planck Institute of Quantum Optics, Garching, Germany

Most of the applications of frequency combs were initially performed using tabletop systems. However, chip-based nonlinear photonics has offered a solution to miniaturization. The tight confinement of light in a waveguide, due to the high refractive index contrast between the core and the cladding of the waveguide, offers high optical nonlinearities and the ability to perform strong dispersion engineering over a broad range of pump wavelengths. Two remarkable on-chip processes that can produce frequency combs with broad spectra, supercontinuum generation in optical waveguides and Kerr comb generation in microresonators, are detailed in a Review by Gaeta, Lipson and Kippenberg. On-chip frequency comb devices that are highly compact, portable, robust and fully integrated with low-power operation are expected to be adopted for a broad range of applications and environments.

Nathalie Picqué and Theodor Hänsch provide a summary of developments in the emerging and rapidly advancing field of atomic and molecular broadband spectroscopy with frequency combs. Their Review focuses on the impact of frequency combs in spectroscopy, where the frequency combs are used to directly excite or interrogate a sample, rather than as a frequency ruler. They explicitly concentrate on a selection of comb synthesizers and techniques where the comb structure is exploited. Spectrometric techniques for frequency comb spectroscopy are described, including direct frequency comb spectroscopy, Ramsey comb spectroscopy, spectroscopy using a dispersive spectrometer, Michelson-based Fourier transform spectroscopy and dual-comb spectroscopy (pictured: two optical comb generators with slightly different, wellchosen line spacing may beat on a detector and generate a radio-frequency spectrum, enabling high-resolution dual-comb spectroscopy). The potential of frequency comb spectrocopy towards the extreme ultraviolet and broadband detection, and an on-chip spectroscopy laboratory are also discussed.

Quantum science requires progressively more complex and large-scale quantum resources. Because of their ability to provide a very high number of temporal and frequency modes that facilitate large-scale quantum systems, frequency combs offer an intriguing solution, and could prove invaluable for a practical and scalable framework of quantum signal and information processing. Michael Kues and colleagues discuss in their Review quantum frequency combs that operate via photon entanglement, beginning with mode-locked quantum frequency combs and followed by energy-time entanglement methods. They discuss the use of photonic integration and fibreoptic telecommunications components in controlling the quantum state, and the potential of these 'quantum microcombs' for photonics-based quantum science.

Frequency combs did not reach their current form until nearly 20 years after the experiments of Hänsch in the late $1970 \mathrm{~s}^{2}$. Several new avenues of research have been explored since then. It will be interesting to see if there will be new branches and surprises enabled by frequency combs in the coming decade.

Published online: 21 February 2019 https://doi.org/10.1038/s41566-019-0388-4 